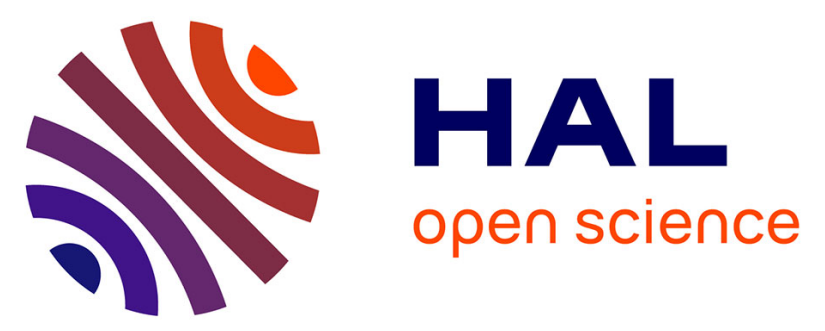

\title{
Heterologous expression of a plant uracil transporter in yeast: improvement of plasma membrane targeting in mutants of the Rsp5p ubiquitin protein ligase.
}

Marine Froissard, Naïma Belgareh-Touzé, Nicole Buisson, Marcello Desimone, Wolf B. Frommer, Rosine Haguenauer-Tsapis

\section{To cite this version:}

Marine Froissard, Naïma Belgareh-Touzé, Nicole Buisson, Marcello Desimone, Wolf B. Frommer, et al.. Heterologous expression of a plant uracil transporter in yeast: improvement of plasma membrane targeting in mutants of the Rsp5p ubiquitin protein ligase.. Biotechnology Journal, 2006, 1(3), pp.308320. hal-00020527

\section{HAL Id: hal-00020527 https://hal.science/hal-00020527}

Submitted on 13 Mar 2006

HAL is a multi-disciplinary open access archive for the deposit and dissemination of scientific research documents, whether they are published or not. The documents may come from teaching and research institutions in France or abroad, or from public or private research centers.
L'archive ouverte pluridisciplinaire HAL, est destinée au dépôt et à la diffusion de documents scientifiques de niveau recherche, publiés ou non, émanant des établissements d'enseignement et de recherche français ou étrangers, des laboratoires publics ou privés. 
1 Heterologous expression of a plant uracil transporter in yeast: improvement of 2 plasma membrane targeting in mutants of the Rsp5p ubiquitin protein ligase

3 Froissard $\mathrm{M}^{\&}$, Belgareh-Touzé $\mathrm{N}^{\&}$, Buisson $\mathrm{N}^{\&}$, Desimone $\mathrm{M}^{\circ}$, Frommer $\mathrm{WB}^{\$}$ and 4 Haguenauer-Tsapis $R^{\AA_{*}}$.

$5 \quad \&$ Institut Jacques Monod-CNRS, Université Paris VI and Paris VII 2 place Jussieu

675251 Paris Cedex 05 France

7 P Plant Physiology, ZMBP, Auf der Morgenstelle 1, D-72076 Tübingen, Germany.

$8 \quad$ Carnegie Institution of Washington, Plant Biology, Stanford, California 94305-4101, 9 USA.

10 Running title: Functional expression of a plant transporter in yeast

$11{ }^{*}$ Corresponding author:

12 Tel: 33144276386

13 Fax: 33144275994

14 E mail: haguenauer@ijm.jussieu.fr

15

16 Keywords: transporters, yeast, heterologous expression, Rsp5p, ubiquitin protein 17 ligase 


\section{Abstract:}

Plasma membrane proteins involved in transport processes play a crucial role in cell physiology. On account of these properties, these molecules are ideal targets for development of new therapeutic and agronomic agents. However, these proteins are of low abundance, which limits their study. Although yeast seems ideal for expressing heterologous transporters, plasma membrane proteins are often retained in intracellular compartments. We tried to find yeast mutants potentially able to improve functional expression of a whole set of heterologous transporters. We focused on Arabidopsis thaliana ureide transporter 1 (AtUPS1), previously cloned by functional complementation in yeast. Tagged versions of AtUPS1 remain mostly trapped in the endoplasmic reticulum and were able to reach slowly the plasma membrane. In contrast, untagged AtUPS1 is rapidly delivered to plasma membrane, where it remains in stable form. Tagged and untagged versions of AtUPS1 were expressed in cells deficient in the ubiquitin ligase Rsp5p, involved in various stages of the intracellular trafficking of membrane-bound proteins. $r s p 5$ mutants displayed further plasma membrane stabilization of untagged AtUPS1, and improved steady state amounts of tagged versions of AtUPS1. rsp5 cells are thus powerful tools to solve the many problems inherent in heterologous expression of membrane proteins in yeast, including ER retention. 


\section{Introduction:}

Systematic sequencing of the genomes of complex organisms (e.g., the plant Arabodopsis thaliana, certain parasites, and man) has revealed a great number of genes that are likely to code for membrane transporters [1]. Many of these proteins are assigned to this functional category on the sole basis of sequence similarity to known transport proteins. Their biochemical properties are thus unknown and information regarding substrate specificity is often lacking. To possess such knowledge would be important both scientifically and industrially, because transport proteins (channels, pumps, and carriers) play a crucial role in cell physiology. This is illustrated by the numerous genetic diseases that are caused by defective transport systems. Furthermore, cell surface membrane transporters may be ideal targets for the development of new therapeutic or agronomic agents. Biological systems are thus required for the functional expression of each transport protein and for the study of its biochemical properties by means of quick, simple tests. The yeast Saccharomyces cerevisiae expression system best meets these requirements. It is easy to use and inexpensive, its "transportome" has been extensively analyzed in silico $[2,3]$ and the function of over 150 yeast transport proteins has been identified [4]. Moreover, the construction of a complete collection of strains deleted for each of the 6200 yeast genes [5] led to an impressive number of $S$. cerevisiae mutants deficient in the transport of compounds as diverse as inorganic ions, metabolites, and drugs. The phenotypes of these mutants are easily identified after growth on solid media and have been used extensively in complementation tests, notably to clone and characterize heterologous transporters. 
Hundreds of plant transporters have been successfully cloned by complementation in yeast [6-9].The yeast system has also been used in biochemical approaches, for example, to analyze the functional properties of plant $\mathrm{H}^{+}$-ATPases [10]. Nevertheless, although yeast seems ideal for expressing plasma membrane proteins including transporters and receptors, investigators often found some of these proteins to be inactive in yeast, sometimes because the protein did not fold properly and/or was not delivered to the plasma membrane but accumulated in a secretory compartment (endoplasmic reticulum (ER), Golgi apparatus, secretory vesicles), sometimes even leading to formation of karmellae [11]. On the other hand, some heterologous proteins correctly delivered to the plasma membrane have been described to undergo rapid endocytosis and turnover, resulting in low plasma membrane levels [12].

A few attempts to improve functional expression in yeast of heterologous plasma membrane transporters have been reported, but they often focused on only one given transporter $[13,14]$. Attempts to identify yeast mutants that would potentially improve the functional expression of a whole set of plasma membrane proteins and more specifically transporters, are still lacking. Finding such mutants for improvement of the functional expression of several plant and mammalian transporters was the objective of an European network, EFFEXPORT ("Engineering yeast for efficient expression of heterologous membrane transporters"). We report here data we obtained within EFFEXPORT in the case of the Arabidopsis Thaliana ureide transporter (AtUPS1), already known to be functionally expressed in $S$. cerevisiae [15]. AtUPS1, was identified by functional complementation of a yeast dal4 dal5 mutant [15] defective in uptake of allantoin. AtUPS1 belongs to a superfamily of plant transporters with five members in Arabidopsis. Analysis of hydrophobicity 
87 predicts 10 putative transmembrane domains, with $\mathrm{N}$ - and $\mathrm{C}$-termini predicted to protrude into the extracellular space. UPS proteins display a conserved central domain between predicted transmembrane helices 5 and 6 , which contains a consensus sequence for a $P$ loop, also designated as a « Walker A » motif for ATP binding [15]. Expression in yeast and Xenopus oocytes allowed to demonstrate that AtUPS1 mediates uptake of allantoin and related metabolites including uracil $[15,16]$. The fate of AtUPS1 can thus be compared to that of the endogenous uracil permease (Fur4p), a well-known yeast transporter, the trafficking of which has been studied extensively (reviewed in [17]). Fur4p, which belongs to a family of five homologous proteins in S. cerevisiae [2], also consists of ten transmembrane spans, with cytoplasmic oriented $\mathrm{N}$ - and $\mathrm{C}$ - termini [18]. Like most yeast plasma membrane proteins, it displays plasma membrane ubiquitylation, catalyzed by the Rsp5p ubiquitin protein ligase, a modification triggering its internalization and subsequent vacuolar degradation [19]. The same ubiquitin ligase, the sole member of the family of Nedd4 ubiquitin ligases in yeast [20], was also demonstrated to be required for direct Golgi-to-vacuole trafficking of a number of plasma membrane transporters, including Fur4p $[17,21]$, i.e. proteins routed to a direct degradation pathway bypassing the plasma membrane under certain nutrient/substrate conditions. Rsp5p was also described to be required for Golgi-to-vacuole traffic of misfolded plasma membrane proteins misrouted to the vacuole [22]. In latter two cases, these proteins were retargeted to plasma membrane in rsp5 mutants. rsp5 mutants thus display increased steady state amounts of many plasma membrane transporters (reviewed in [17]), and were good candidates for potential improvement of functional expression of heterologous transporters. However, in addition to its role in trafficking of membrane proteins [17], Rsp5p has many other functions, including essential 
112 functions $[23,24]$. Hence, only defined rsp5 mutants may be used for optimization of

113 functional expression of heterologous proteins. Two viable rsp5 mutants that were

114 described to be affected for several of the trafficking functions of Rsp5p appeared

115 interesting: npi1 mutant, with altered RSP5 promoter, leading to a 10-fold reduction in

116 the steady state amount of this protein [25], and rsp5 $\Delta C 2$, lacking Rsp5p C2 domain

117 involved in localization of the enzyme at plasma membrane and endosomes [26].

We report here an analysis of the fate of AtUPS1, as compared to that of

119 Fur4p, in wild type and rsp5 mutant cells, and show that npi1 and rsp5 4 C2 cells

120 improved functional expression of the plant transporter.

\section{Results}

\section{AtUPS1 mediates high affinity uracil transport in yeast}

As outlined above, when expressed in yeast, AtUPS1 transports [ ${ }^{14} \mathrm{C}$ ] labelled allantoin with high affinity and potentially other heterocyclic compounds as suggested by competition studies [15]. Moreover, AtUPS1 mediates uracil uptake when expressed in Xenopus oocytes [16]. In order to monitor the intracellular fate of

129 AtUPS1 in yeast, UPS1 was cloned in a multicopy plasmid under the control of the 130 galactose-inducible GAL promoter. We defined the characteristics of uracil uptake

131 mediated by GAL-UPS1, as compared to uracil uptake mediated by the endogenous

132 Fur4p cloned under the control of the same promoter. fur4 $\Delta$ cells grown on galactose

133 expressing either GAL-UPS1 or GAL-FUR4 displayed high sensitivity to 5-fluorouracil 134 (5FU), a toxic analog of uracil: cells expressing the transporters were unable to grow 135 on plates containing $1 \mu \mathrm{M} 5 \mathrm{FU}$, whereas cells transformed with a control plasmid 
136 grew normally (Fig 1A). To determine the uracil transport properties of AtUPS1

137 quantitatively, radiotracer uptake studies were performed using $\left[{ }^{14} \mathrm{C}\right]$ uracil. $\left[{ }^{14} \mathrm{C}\right]$

138 uracil uptake mediated by GAL-UPS1 expressed in fur4 $\Delta$ cells growing exponentially 139 and fully induced on galactose was linear for at least 3 min, concentration-

140 dependent, and displayed saturation kinetics with an apparent $\mathrm{Km}$ of $6 \mu \mathrm{M}$, close to 141 that observed in parallel for cells expressing GAL-FUR4 (7.5 $\mu \mathrm{M})$. fur4A cells 142 expressing GAL-UPS1 grown overnight in galactose containing media displayed an 143 activity $50 \%$ of cells expressing GAL-FUR4, i.e., more than 30 -fold that of 144 chromosomal encoded FUR4 (Fig. 1B), thus providing a sensitive assay to follow 145 AtUPS1 intracellular fate.

Insight into AtUPS1 intracellular trafficking in yeast Inducibility of AtUPS1 synthesis after galactose induction provides a useful

149 tool for monitoring plasma membrane delivery, as previously demonstrated for the

150 yeast Fur4p [27]. We measured uracil uptake activity after galactose induction of

151 GAL-UPS1 and GAL-FUR4 in fur4A cells in parallel (Fig. 2A). In both cases, activity,

152 as a measure of plasma membrane targeting, was detectable after 30 min induction.

153 The increase in uracil uptake activity was linear for one hour with a similar slope for 154 cells expressing the yeast and the plant transporters. Hence the heterologous 155 AtUPS1 appears to be recognized efficiently by the yeast secretory machinery. We 156 then compared the fate of AtUPS1 and Fur4p along the endocytic pathway using an 157 experimental condition known to trigger rapid Fur4p internalization and subsequent 158 vacuolar degradation, i. e. the inhibition of protein synthesis by addition of 159 cycloheximide [28] (Fig. 2B). Cycloheximide was added to exponentially growing 160 fur4 $\Delta$ cells induced overnight in galactose for expression of either AtUPS1 or Fur4p. 
161 Uracil uptake activity of cells expressing Fur4p decreased rapidly ( $t_{1 / 2}=45 \min$ ),

162 whereas uracil uptake activity of cells expressing AtUPS1 did not display a decrease

163 for four hours. Hence, once delivered to the plasma membrane, the plant AtUPS1

164 was remarkably stable, and did not undergo obvious endocytosis after inhibition of 165 protein synthesis.

\section{C-terminally tagged versions of AtUPS1 are retained in the ER}

The monitoring of uracil uptake activity can afford insight into the intracellular

fate of AtUPS1, since it provides information about the plasma membrane located

170 transporter. This information, however, remains limited, and does not indicate the

171 fraction of plasma membrane-delivered protein versus potential intracellular pools. In

172 the absence of available antibodies, we decided to monitor the fate of tagged

173 versions of AtUPS1. C-terminally tagged versions of AtUPS1 were constructed, first

174 with a GFP-tag, a powerful tool for monitoring the intracellular fate in yeast of plasma

175 membrane proteins of heterologous [14] or endogenous origin, including that of

176 Fur4p [29-31]. fur4 4 cells transformed with a multicopy plasmid carrying GAL-UPS1-

177 GFP displayed high 5FU sensitivity after growth on galactose (Fig. 3A). Strikingly,

178 fur4 $\Delta$ cells transformed with multicopy plasmid carrying GAL-UPS1 tagged with the

179 smaller HA epitope displayed intermediate 5FU sensitivity after growth on galactose,

180 indicating that a smaller tag did not improve AtUPS1 functionality. According to these

181 plate assays, C-terminally tagged AtUPS1 thus appeared functional, notably GFP-

182 tagged AtUPS1. However, fur4A cells transformed by the multicopy plasmid carrying

183 GAL-UPS1-GFP and fully induced displayed only a very low level of uracil uptake 184 activity $\left(0.04 \mathrm{nMol} / \mathrm{min} / \mathrm{A}_{600}\right)$, an activity 50 fold lower than that observed in the case

185 of induced cells transformed by a plasmid carrying untagged AtUPS1 (not shown). 
186 The use of a smaller tag (HA) or another promoter (CYC1) did not improve uracil 187 uptake activity.

188 The GFP -or HA- tags may inhibit transport activity or impair plasma 189 membrane delivery of tagged transporter due to folding problems. We checked GFP

190 fluorescence in time course experiments using the multicopy plasmid carrying the 191 GAL-UPS1-GFP (Fig. 3B), or the multicopy plasmid GAL-Fur4-GFP as a control.

192 Galactose induction of Fur4-GFP led, after $30 \mathrm{~min}$, to observation of small internal 193 compartments, likely Golgi/secretory vesicles. 30 min later, plasma membrane 194 staining was clearly evidenced, often in a polarized fashion, with intense staining of 195 small buds (Fig. 3B). The distribution of AtUPS1-GFP was strikingly different. After $19630 \mathrm{~min}$ of galactose induction of AtUPS1-GFP expression, a perinuclear staining was 197 clearly observable. 30 min later, or after overnight induction, the perinuclear staining 198 was still present and a discontinuous staining at/or underneath the plasma 199 membrane has appeared. This pattern is typical of the yeast ER. Intense staining of 200 lines or spots, as if intracellular membranes had formed aggregates were also 201 visualized (Fig. 3B). Indeed, electron microscopy of the ultrastructural morphology of 202 cells fully induced for expression of AtUPS1-GFP revealed proliferation, hanks of ER 203 membranes (Fig. 3D), as often described in the case of cells overexpressing ER204 retained proteins [11]. This altered morphology did not resulted from the 205 overexpression of a membrane protein as such: overnight overexpression of 206 endogenous FUR4 from the same multicopy, GAL-inducible plasmid, did not lead to 207 altered morphology (not shown).

208 ER-retention of GFP-tagged AtUPS1 apparently did not result from the mere 209 overexpression of this protein. Perinuclear/ER staining was also evidenced upon 210 expression of AtUPS1-GFP from a centromeric plasmid under the control of the mild 
211 strength CYC1 promoter, leading to a steady state protein level about 4-fold lower

212 than that observed after two hours galactose induction of GAL-UPS1-GFP (Fig. 3B

213 and C). Furthermore, when analyzed by sucrose gradient fractionation, AtUPS1-GFP

214 expressed from the CEN CYC1-plasmid or from the $2 \mu$ GAL-inducible plasmid 215 displayed exactly the same pattern with major pool in internal fractions (data not 216 shown).

217 Hence, the GFP tag at the C-terminus of AtUPS1 obviously triggers ER 218 retention of the transporter. The low uracil uptake activity of cells expressing this 219 transporter can only be observed after long expression periods (3-4 hours, cf Fig. 220 6B), and likely corresponds to the low fraction of protein finally reaching the plasma 221 membrane. With such folding problems, overexpression is a way to increase steady 222 state plasma membrane expression: both 5FU sensitivity and uracil uptake activity 223 were improved in the case of AtUPS1-GFP expression from a multicopy plasmid and 224 strong GAL promoter compared to expression from a centromeric plasmid under the 225 control of a lower strength promoter.

Intracellular fate of AtUPS1 carrying an internal myc tag in yeast: slow ER exit but final plasma membrane delivery

We checked whether insertion of a small myc-tag inside the central loop 230 containing the Walker A motif would be a better way to study AtUPS1 intracellular 231 trafficking. Cells expressing internally myc tagged AtUPS1 from a multicopy 232 galactose inducible plasmid displayed high sensitivity to 5FU (Fig. 4A). Cells 233 transformed with the multicopy GAL-UPS1 ${ }^{m y c}$ plasmid grown overnight in galactose 234 displayed a relatively high level of uracil uptake activity: $1 \mathrm{nMol} / \mathrm{min} / \mathrm{A}_{600}$, i. e. $70 \%$ 235 that observed in the case of untagged AtUPS1 (Fig. 4B), suggesting that the myc tag 
236 at this position had only a small impact on AtUPS1 function. However, this tag had a 237 clear influence on AtUPS1 intracellular trafficking. Induction experiments showed that 238 uracil uptake activity appeared slowly: 4-6 hours were necessary before we were 239 able to measure any detectable uptake activity (Fig. 4C). Aliquots withdrawn at

240 several time points after galactose induction were analyzed by protein gel blots using 241 a specific anti-myc antibody. AtUPS1 ${ }^{\text {myc }}$ appeared on gels as a band of apparent

242 molecular mass of about $36 \mathrm{kDa}, i$. e. slightly below the expected molecular mass 243 deduced from predicted protein sequence (44 kDa) (Fig. 4E), as often observed for

244 very hydrophobic proteins. A comparable pattern was observed after overnight galactose induction of AtUPS $1^{\text {myc }}$ expressed from either a centromeric or a multicopy 246 plasmid (data not shown).

In order to check the intracellular location of AtUPS $1^{\text {myc }}$ derived species, cells

248 transformed with GAL-UPS1 ${ }^{m y c}$ were induced by addition of galactose, aliquots 249 withdrawn periodically were fixed and analyzed by immunofluorescence using a 250 monoclonal anti-myc antibody. One hour induction was sufficient to observe a 251 specific signal, mostly perinuclear, supported by simultaneous DAPI staining (Fig.

$2525 \mathrm{~A}$ ). To obtain better resolution of the cell surface staining (potentially corresponding 253 to either ER or plasma membrane), cells were examined by confocal microscopy. 254 The lower background in optical slices made it possible to show that all cells 255 displayed perinuclear staining, together with discontinuous regions of cell surface 256 staining (Fig. 5B). Because cell surface was not homogeneously stained, we can 257 conclude that the main signal corresponds to ER staining and that a plasma 258 membrane localisation cannot be detected by this approach. The low rate of uracil 259 uptake activity thus likely resulted from low ER exit rates (Fig. 4C) The finding that 260 the activity of fully induced cells is in a similar range as that of cells expressing 
261 untagged AtUPS1, which was rapidly targeted to plasma membrane, may suggest 262 that after slow rates of ER exit, AtUPS $1^{\text {myc }}$ finally reached the plasma membrane. 263 Further indication that AtUPS $1^{\mathrm{myc}}$ stored in internal compartments could finally reach 264 plasma membrane was provided by the observation of some increase in uracil 265 uptake activity after stopping transporter synthesis by the addition of CHX (Fig. 4D). 266 Once targeted at the plasma membrane, AtUPS $1^{\text {myc }}$ was rather stable, as judged 267 from the extreme stability of uracil uptake activity for over 3 hours after this transient 268 uracil uptake activity increase following $\mathrm{CHX}$ addition. However, some low rate 269 endocytosis likely occurred as compared to the incredibly stable untagged AtUPS1 270 (Fig. 4D).

Mutations in the Rsp5 ubiquitin protein ligase improve functional expression of tagged and untagged AtUPS1 in yeast

Despite the difficulties encountered in tagging AtUPS1 at different positions, 276 the high sensitivity of the two functional tests, 5FU sensitivity and uracil uptake 277 measurements, provided suitable tools to check whether yeast mutants could 278 improve the steady state levels of functional tagged or untagged AtUPS1 versions. 279 We used two viable rsp5 mutants, npi1 and rsp5 $\Delta C 2$, that display delayed 280 endocytosis of several cargoes, including Fur4p. Accordingly, they displayed 281 increased 5FU sensitivity as a result of the plasma membrane stabilization of 282 endogenous chromosomal or plasmid encoded Fur4p (not shown). We checked the 283 fate of tagged and untagged versions of AtUPS1 in npi1 and rsp5 $\triangle$ C2 mutant cells.

284 For this purpose, FUR4 was deleted in wild type, npi1 and rsp5 4 C2 cells, and 285 the resulting strains were transformed with multicopy plasmids carrying either UPS1 
286 under the control of the constitutive PGK promoter (plasmid pFL61-UPS1), or 287 galactose inducible UPS1, UPS1-GFP and UPS $1^{\text {myc }}$. Transformed cells grown in 288 glucose, or galactose containing media in the case of plasmids with a GAL promoter 289 displayed identical growth in the absence of 5FU (Fig. 6A), indicating that neither the 290 mutations, nor the expression of the plant transporters impaired growth. 5FU 291 sensitivity of transformants was strikingly enhanced in both rsp5 $\triangle C 2$ and npi1 cells 292 expressing the tagged and untagged plant transporter when compared to wild type 293 cells, with slightly stronger effect promoted in all cases by the npi1 mutation 294 (compare the size of isolated colonies) (Fig. 6A). This suggests higher amounts of 295 plasma membrane tagged or untagged transporters in the mutants. In the case of the 296 untagged transporters, the enhancement in 5FU sensitivity was evidenced after 297 synthesis from PGK or GAL10 promoter at different 5FU concentrations (Fig. 6A), 298 indicating that $r s p 5$ mutations interfered with trafficking, rather than with galactose299 driven expression. For untagged AtUPS1 which is rapidly delivered to the plasma 300 membrane and very stable in wild type cells, the increase in functional transporter 301 activity observed in rsp5 amutants may result from inhibition of some direct Golgi to 302 vacuole targeting, or from protection against a possible low basal endocytosis, for 303 instance when cells reached stationary phase, a likely situation for cells grown 304 several days on plates.

In the case of the myc-tagged version of AtUPS1, we monitored the fate of the 306 transporter after galactose induction by western blots (Fig 6C), and uracil uptake 307 measurements (Fig. 6B). In agreement with fluorescent data, AtUPS1 ${ }^{\text {myc }}$ protein was 308 already detectable in wild type cells at early time points after induction (30-60 $\mathrm{min}$ ) 309 but uracil uptake was readily measurable only at far later time points (4 hours). In npi1 cells, AtUPS $1^{\text {myc }}$ was detectable in higher amounts at all time points and uracil 
311 uptake activity appeared at least one hour earlier compared to wild type cells (Fig.

$3126 \mathrm{~B})$. rsp5 $\Delta C 2$ mutation also resulted in more rapid appearance of uracil uptake

313 activity. Strikingly, even if the activity was lower for cells expressing AtUPS1-GFP

314 compared to AtUPS1 ${ }^{\text {myc }}$, the rate of appearance of uptake was very similar. For both

315 types of tagged transporters uracil uptake activity was thus 2-3 fold higher in mutant 316 cells than in wild type cells after 4 hour induction. Since the major pool of both

317 AtUPS1-GFP and AtUPS $1^{\text {myc }}$ seemed to be in the ER, both npi1 and rsp5 42

318 mutations apparently lead to accelerated ER exit.

Discussion:

The use of a regulable promoter and the high sensitivity of uracil uptake

322 measurements enabled us to obtain crucial information about the fate of untagged

323 AtUPS1 uracil transporter in yeast. AtUPS1 is rapidly delivered to the plasma

324 membrane, with kinetics indistinguishable from those of endogenous yeast

325 transporter. Hence, the yeast secretory pathway efficiently handles the heterologous

326 plant transporter. It is possible that such property was the reason for the successful

327 cloning by functional expression of so many plant transporters when using yeast as a

328 host system [6,7]. Plasma membrane delivered AtUPS1 exhibited striking stability,

329 notably when compared to the endogenous yeast uracil permease especially 330 susceptible to stress-induced endocytosis [28]. This was true for both untagged

331 transporter and for internally tagged AtUPS $1^{\text {myc }}$ (not shown). This differential stability

332 between yeast and plant uracil transporters may possibly reflect fundamental

333 differences in their endocytic processes. Endocytosis in yeast is dependent on prior

334 ubiquitylation of plasma membrane cargoes by the Rsp5p ubiquitin protein ligase

335 [17]. A subset of mammalian proteins undergo ubiquitin-dependent endocytosis, 
sometimes involving ubiquitin-protein ligases of the Nedd4/Rsp5 family [32]. In contrast, although plants display numerous ubiquitin protein ligases, they do not seem to have Rsp5p orthologs [33]. The extreme stability of AtUPS1 in yeast could result from the absence or low accessibility of ubiquitylation sites that Rsp5p can recognize, at least under the experimental conditions we tested. One possible reason could be the lumenal orientation of both AtUPS1 $\mathrm{N}$ - and C-termini.

In addition to obtaining information on intracellular trafficking of the plant AtUPS1 in yeast based on uptake measurements, we tried to gain new insights into the biochemical properties of this transporter. Unfortunately, tagging the transporter, either at the N-terminus (not shown) and C-terminus or inside its central loop, lead to folding problems, often resulting in ER retention of most of the protein, preventing us from using these tagged versions to obtain a judicious biochemical characterization of this transporter. A marked difference, however, was observed between internal tagging, and tagging at the $\mathrm{N}$ - and $\mathrm{C}$-termini, which was far more deleterious. Internally tagged transporter displayed delayed ER exit, but finally reached the plasma membrane in a fully functional state, and fully induced cells expressing this version of the transporter displayed activity closely resembling that of cells expressing untagged transporter. In contrast, cells expressing C-terminally tagged transporter displayed 40-fold less uracil uptake activity compared to cells expressing untagged transporters, probably because of a very low percentage of the protein exiting the ER. In latter case, some improvement could be achieved by increased levels of expression (stronger promoter, multicopy versus centromeric plasmid). The deleterious effect of C-terminal tags could result from the unusual structure of the transporter. Hydrophobicity plot analysis of proteins of the UPS family suggested an external orientation of both termini [15] a situation somewhat rare for transporters. 
361 Most yeast transporters, for instance, display cytoplasmic oriented termini, as

362 predicted from hydrophobicity plot analysis or from experimental data [2]. Many of

363 these tranporters were studied after tagging, most often at their C-terminus, a

364 modification that did not induce any major trafficking problems. The observation that

$365 \mathrm{~N}$ - and C-terminal tags impaired AtUPS1 folding is compatible with an external

366 orientation, but experimental data are required to prove this. One obvious lesson

367 from these observations is that tagging at the positions and with the tags used in the 368 present study may probably influence the fate of UPS proteins in plant cells in a 369 similar way as in yeast cells. Indeed, previous studies attempting to study the 370 subcellular location of $\mathrm{N}$ - or C-terminal fusions of UPS proteins with GFP by transient 371 expression in Arabidopsis protoplasts showed that these fusion proteins did not 372 reach the plasma membrane (Schmidt, A., Baumann, N. and Desimone, M., 373 unpublished data). This suggests that yeast may also represent a useful 374 experimental system to decipher where tags can be introduced in heterologous 375 transporters.

376 The distinctive behaviour of the various versions of AtUPS1, one correctly 377 targeted to the plasma membrane, and two retained in the ER to various extents, 378 provided the opportunity to check how to improve the steady state plasma membrane 379 amount of heterologous transporters with distinct intracellular fates. We checked 380 specifically the influence of mutations in Rsp5p. This ubiquitin protein ligase is 381 involved in various stages of intracellular trafficking of membrane-bound proteins, 382 including ER-associated degradation, plasma membrane internalization, Golgi to 383 vacuolar trafficking, and sorting to multivesicular bodies [17]. Limiting or preventing 384 latter three trafficking steps results in elevated steady state levels of yeast 385 transporters. Two specific viable mutants were used, npi1, with decreased amounts 
386

387

of Rsp5p [25] and rsp5 4 C2, lacking the C2 domain of Rsp5p [34], dispensable for viability, but critical for Rsp5p trafficking functions [26,34-38]. Strikingly, both mutants improved steady state amounts of functional, plasma membrane targeted, tagged and untagged versions of AtUPS1, as judged from increased 5FU sensitivity. In the case of untagged transporter, increased 5FU sensitivity was observed after expression of the transporter under the control of the constitutive PGK promoter, or the inducible GAL10 promoter, indicating that the mutations interfered as expected with trafficking rather than with expression.

In the case of the untagged version of AtUPS1, it was difficult to further explore the mechanism of this improvement. Plasma membrane AtUPS1 was so stable in wild type cells submitted to $\mathrm{CHX}$ treatment that further stabilisation in rsp5 cells could not be detected in this type of experiment. The improvement in plasma membrane steady state amounts of AtUPS1 in npi1 and rsp5 4 C2 cells might possibly arise from a low basal endocytosis of AtUPS1, undetectable in CHX chase experiments, but possibly occurring once cells reach stationary phase, as is the case for cells grown on plates. Alternatively, the increase in plasma membrane AtUPS1 in these rsp5 mutants could result from a decreased direct Golgi to vacuole targeting of a fraction of the transporter.

Mutations in RSP5, either npi1 or rsp5 $4 C 2$, had a similar impact on the intracellular fate of tagged versions of AtUPS1 at both the C-terminus or in the intracellular loop. These two variants of AtUPS1 were mainly located in the ER, with possibly exit in only very limited amounts (AtUPS1-GFP), or more important final amounts (AtUPS $1^{\text {myc }}$ ) thus leading to uracil uptake activity similar to that displayed by cells expressing untagged transporters. In both cases, the rate of plasma membrane delivery of uracil uptake activity was greatly delayed, with activity measurable after 3- 
4114 hours induction instead of $30 \mathrm{~min}$ for AtUPS1 -i. e. long after detection of the

412 protein on Western blots- and the two rsp5 mutations reduced this delay. At first

413 glance, this effect can be attributed to an effect of Rsp5p on ER exit. Among its many

414 functions, Rsp5p was described to be involved in ubiquitylation followed by

415 proteasome degradation of several misfolded soluble and membrane-bound proteins

416 retained in the ER, notably under conditions of saturation of other ER-associated

417 degradative (ERAD) pathways [39]. Tagged forms of AtUPS1 might be partly

418 susceptible to such an Rsp5p-dependent ER-associated degradation, and inhibition

419 of this degradation in npi1 and rsp5 $\Delta C 2$ mutants would result in more rapid ER exit of

420 transporters escaping this degradation to some extent. It is also possible that a small

421 number of misfolded tagged transporters undergo ER to Golgi trafficking, followed by

422 Golgi to vacuole sorting. Rsp5p was also shown to be involved in Golgi to vacuole

423 trafficking of some misfolded mutant plasma membrane proteins [22] or of yeast

424 transporters displaying direct vacuolar targeting under specific nutrient conditions

425 [17]. In both cases in rsp5 mutants, these proteins are directed to plasma membrane

$426[22,40]$. A small fraction of tagged AtUPS1 could undergo such direct vacuolar

427 trafficking, a process inhibited in rsp5 mutants. In support of a partial direct Golgi to

428 vacuole trafficking of AtUPS $1^{\text {myc }}$, we observed that pep4 mutant cells, deficient for

429 vacuolar protease activities, displayed higher amounts of the $36 \mathrm{kDa}$ AtUPS1 ${ }^{\text {myc }}$

430 species than wild type cells even after short induction times, as did vps23 $\Delta$ mutants,

431 impaired in Golgi to vacuole trafficking (data not shown). The increase in plasma

432 membrane amounts of functional forms of tagged AtUPS1 in rsp5 mutants, attested

433 in exponentially growing cells by increased uracil uptake activity, and on plates by

434 increased 5FU sensitivity might also result from an inhibition of Rsp5p-dependent 
435 processes at three levels: ERAD, Golgi-to-vacuole trafficking and plasma membrane 436 internalization.

437 Rsp5p plays a key role in trafficking of yeast plasma membrane proteins. The 438 present study shows that several viable rsp5 mutants display increased plasma 439 membrane amounts of a plant transporter, rapidly targeted to the plasma membrane. 440 Strikingly, within the same european network (EFFEXPORT), other laboratories 441 observed, in viable rsp5 mutants, improved functional expression of several 442 heterologous transporters, including several $\mathrm{NH}_{4}{ }^{+}$transporters ( $\mathrm{Rh}$ family) of animal 443 origin (Marini $\mathrm{A}$ and André $\mathrm{B}$, personal communication), and increased plasma 444 membrane amounts versus internal fractions of mammalian $\mathrm{Na}^{+} / \mathrm{H}^{+}$antiporters 445 (Flegegova, H, Haguenauer-Tsapis, R and Sychrova, H. Biochem. Biophys. Acta, in 446 press). In addition, these mutants optimize plasma membrane delivery of tagged 447 versions of the plant AtUPS1, stacked in the ER as a result of folding problems. ER 448 retention is one of the major problems encountered in the case of expression of 449 heterologous plasma membrane proteins in yeast. The increase in steady state 450 plasma membrane amounts of heterologous plant and mammalian transporters, 451 upon expression in viable rsp5 mutants, show that these cells could be powerful tools 452 to solve the many problems inherent in heterologous expression of membrane 453 proteins, including ER retention.

\section{Materials and methods}

Yeast strains and growth conditions

458 Yeast strains were transformed as described by Gietz et al [41]. Cells were grown at 459 $30^{\circ} \mathrm{C}$ in minimal medium (YNB) containing $0.67 \%$ yeast nitrogen base without amino 
460 acids (BD bioscience, NJ, USA), and supplemented with appropriate nutrients. The 461 carbon source was $2 \%$ glucose, or $2 \%$ galactose plus $0.05 \%$ glucose as indicated in 462 the figure legends. Galactose induction was performed on cells grown overnight in $4632 \%$ raffinose plus $0.05 \%$ glucose up to an $A_{600 n m}=0.5$. Galactose $(2 \%)$ was then 464 added to the medium.

465 The disruption of FUR4 gene was achieved by ORF replacement with long flanking 466 homology regions to the KanMX4 cassette corresponding to the strategy described 467 by Wach [42].

Growth tests in the presence of 5-fluorouracil

470 Cells, prototroph for uracil, were cultured overnight in minimal medium containing 471 glucose and spotted on plates containing minimal medium with galactose to induce expression of FUR4 or UPS1 variants and supplemented with various concentrations of 5FU (Sigma-Aldrich, Lyon, France). The first drop contained $3.10^{4}$ cells and each

474 subsequent drop was diluted six-fold compared to the prior drop.

Construction of plasmids

477 DNA manipulations, including restriction analysis and ligations, were performed 478 essentially as described by Maniatis et al. [43].

479 The control plasmid p195gF-GFP (pRT208) expressing FUR4-GFP under the control 480 of the GAL10 promoter was constructed by cloning a Pstl/BamH1 fragment (FUR4481 GFP) from pFL38gF-GFP [30] into p195gF [28] at the Pstl/BamHI site.

482 The pGAL-UPS1 (pRT205) plasmid was constructed by insertion of a BamHI/EcoRI 483 fragment encoding UPS1 (with pFL61-UPS1 as a template [15]) in the BamHI/EcoRI 484 site of pYEF1 [44]. 
485 To construct the plasmid GAL-UPS1-GFP (pRT206) we first built the pNBT29 486 plasmid, which contains the yeast enhanced GFP under the control of the GAL10 487 promoter on a multicopy plasmid. For this purpose, a BamHI/Notl fragment encoding 488 GFP was obtained by PCR using the pUG35 plasmid as a template [45] and 489 introduced at the BamHI/Notl site of pYEF1 [44]. Then a BamHI/Clal fragment 490 encoding AtUPS1 was amplified by PCR using pFL61-AtUPS1 [15] as a template, and introduced at the BamHI/Clal site of pNBT29 in frame with the coding sequence 492 of GFP thereby creating a GFP C-terminally tagged version of AtUPS1. To construct 493 the CYC1-UPS1-GFP (pRT207) plasmid we cloned the BamHI/EcoRI fragment 494 containing UPS1-GFP at the BamHI/EcoRI site of the p416-CYC1 plasmid [46] .

495 The pGAL-UPS1-HA (pRT204) plasmid was obtained by insertion in the Clal site of 496 the pYEF2 [44], in frame with the coding sequence of the HA tag, of a fragment 497 encoding UPS1 obtained by restriction of the plasmid GAL-UPS1-GFP.

498 To construct the plasmid pGAL-UPS1 ${ }^{m y c}$ (pRT203) two fragments of UPS1 were 499 amplified separately by PCR using pFL61-UPS1 as a template. One fragment 500 contained at start an EcoRI and an Xbal site, the 5'-portion of the UPS1 coding 501 sequence (from ATG to the position 549) and a BamHI site at the end. The other 502 fragment contained a BamHI site at the start, the coding sequence for the c-myc 503 epitope, the 3'- portion of UPS1 (from position 550 to the stop codon), and an EcoRI 504 and a Xhol site at the end. Both fragments were sequentially cloned in pDR199 [47] 505 using the EcoRI/BamHI sites for the first fragment and BamHI/and Xhol for the 506 second. After sequencing, the complete c-myc tagged UPS1 sequence was obtained 507 by restriction and subcloned into the Xba I / Xho I sites of the CEN plasmid p416508 GAL [46]. Afterwards, the multicopy plasmid pGAL-UPS1 ${ }^{\text {myc }}$ was obtained by 
509 subcloning a Sacl/Xhol fragment of the p416-GAL-UPS $1^{\text {myc }}$ into the Sacl/Xhol sites

510 of the p426-GAL plasmid [46].

511

512 Measurement of uracil uptake.

513 Uracil uptake was measured in exponentially growing cells as previously described.

514 Yeast culture $(1 \mathrm{ml})$ was incubated with $5 \mu \mathrm{M}\left[{ }^{14} \mathrm{C}\right]$ uracil (ICN biomedicals Illkirch,

515 France) for $20 \mathrm{sec}$ at $30^{\circ} \mathrm{C}$, then quickly filtered through Whatman GF/C filters, which

516 were in turn washed twice with ice-cold water and counted for radioactivity. In the

517 case of low uracil uptake activity, this basic protocol was slightly modified, with the 518 use of two $\mathrm{ml}$ samples and incubation for $2 \mathrm{~min}$ at $30^{\circ} \mathrm{C}$.

Michaelis-Menten kinetics

521 Uracil uptake activities measured at various substrate concentrations were fitted to a

522 hyperbola with SIGMA PLOT 5.0, V5 according to Michaelis-Menten kinetics.

Yeast cell extracts, SDS-PAGE and Western immunoblotting

Total protein extracts were prepared by the $\mathrm{NaOH} /$ Trichloroacetic acid (TCA) lysis technique as described in [28]. Proteins were separated by SDS-PAGE on Tricine

527 gels and transferred onto nitrocellulose membranes. The membranes were probed 528 with monoclonal antibodies against GFP (Roche Diagnostics Meylan, France), or 529 myc (9E10 from Roche Diagnostics), or polyclonal antibody against Gas1p (a kind 530 gift from H. Riezman). Primary antibodies were detected using horseradish 531 peroxidase-conjugated anti-rabbit or anti-mouse IgG secondary antibody (Sigma532 Aldrich, Lyon, France) revealed by ECL chemiluminescence (Amersham). 
534 Immunofluorescence

535 Immunofluorescence was performed as described in [48] except that cells were 536 permeabilized with $0.5 \%$ Triton $\mathrm{X} 100$. The primary antibody was the monoclonal anti537 Myc (9E10 from Roche Diagnostics, Meylan, France)and the secondary antibody 538 was an FITC-conjugated goat anti-mouse-IgG (Jackson ImmunoResearch

539 Laboratories, Inc., West Grove, PA). For DNA staining, $1 \mu \mathrm{g} / \mathrm{ml}$ Diamin-Phenylindol540 Dihydrochlorid (DAPI) was used. Samples were viewed under an Olympus 541 microscope BY61 using FITC and DAPI filter sets. Image acquisition was performed 542 using a Spot charge-coupled device camera SPOT4.05 .

543 For confocal analysis, cells were imaged using an inverted microscope (Leica, Inc.

544 Wetzlar, Germany) and scanning was performed with a True Confocal Scanner LEICA 545 TCS 4D.

\section{Electron microscopy}

548 Yeast cells were fixed by adding $200 \mu \mathrm{l}$ of $50 \%$ aqueous glutaraldehyde to $10 \mathrm{ml}$ of 549 growth medium for $10 \mathrm{~min}$ and then centrifuged at $5000 \mathrm{~g}$ for $10 \mathrm{~min}$ at $4^{\circ} \mathrm{C}$. After 550 fixation with fresh fixatives for $2 \mathrm{~h}$ at $4^{\circ} \mathrm{C}$, cells were washed in $0.1 \mathrm{M}$ cacodylate 551 buffer $(\mathrm{pH} 7.4)$ and in water. Subsequently, cells were treated with $1 \% \mathrm{KMnO} 4$ for $2 \mathrm{~h}$ 552 on ice, washed in water and re-suspended in $2 \%$ aqueous uranyl acetate for $1 \mathrm{~h}$ at $5534^{\circ} \mathrm{C}$. Cells were dehydrated in a graded series of ethanol, infiltrated in a mixture of 554 ethanol and Spurr's resin and embedded in Spurr's low viscosity media. Thin 555 sections were cut, stained with lead citrate and examined in a Tecnai 12 electron 556 microscope (Eindhoven, Netherlands). 
TABLE 1. List of strains

\begin{tabular}{|c|c|c|c|}
\hline Strain & Background & Genotype & Source \\
\hline MF04 & $\Sigma 1278 b$ & MATa ura3 trp1 FUR4::KanMX4 & This study \\
\hline MF05 & $\Sigma 1278 b$ & MATa ura3 trp1 rsp5 4 C2 FUR4::kanMX4 & This study \\
\hline MF06 & $\Sigma 1278 b$ & Mata ura3 trp1 npi1 FUR4::kanMX4 & This study \\
\hline $27061 b$ & $\Sigma 1278 b$ & Mata ura3 trp1 & [19] \\
\hline $27064 b$ & $\Sigma 1278 b$ & Mata ura3 trp1 npi1 & [19] \\
\hline BY4741 & BY & 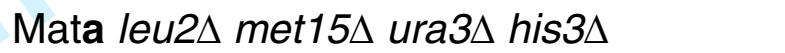 & Euroscarf \\
\hline
\end{tabular}

559

560

TABLE 2. List of plasmids

\begin{tabular}{lcc}
\hline \multicolumn{1}{c}{ Plasmid } & \multicolumn{1}{c}{ Characteristics } & Source \\
\hline pPGK-UPS1 (PFL61-UPS1) & $2 \mu$, URA3 prom. PGK-UPS1 & {$[15]$} \\
\hline pGAL (PYeF2) & $2 \mu$, URA3, prom. GAL10, Cter HA & {$[44]$} \\
pGAL-FUR4 (pFL38gF) & CEN, URA3, prom. GAL10, FUR4 & {$[30]$} \\
p195gF & $2 \mu$, URA3, prom. GAL10, FUR4 & {$[28]$} \\
p195gF-GFP (pRT208) & $2 \mu$, URA3, prom. GAL10-FUR4-GFP & This study \\
pGAL-UPS1-HA (pRT204) & $2 \mu$, URA3, prom. GAL10, UPS1- HA & \\
& & This study \\
pGAL-UPS1 (pRT205) & $2 \mu$, URA3, prom. GAL10, UPS1 & This study \\
pGAL-UPS1-GFP (pRT206) & $2 \mu$, URA3, prom. GAL10, UPS1-GFP & This study \\
pCYC1-UPS1-GFP (pRT207) & CEN, URA3, prom.CYC1, UPS1- & This study \\
& GFP & \\
\hline
\end{tabular}


563

564

565

566

567

568

569

570

571

572

573

574

575

576

577

578

579

580

581

582

583

584

585 of initial activities.

\section{Figure 1: Comparison of uracil uptake activity of Arabidopsis thaliana UPS1} expressed in yeast and endogenous uracil permease Fur4p.

A: 5-Fluorouracil (5FU) sensitivity. fur4 $\Delta$ cells transformed with either pGAL (empty vector), pGAL-UPS1 or pGAL-FUR4 were grown on galactose containing plates supplemented or not with $1 \mu \mathrm{M} 5 \mathrm{FU}$ (toxic analog of uracil).

B: Uracil uptake activity of AtUPS1 compared to Fur4p. fur4A strains transformed with pGAL (white), pGAL-UPS1(grey) or pGAL-FUR4 (black) were grown to exponential phase in galactose containing medium and used for measurement of $\left[{ }^{14} \mathrm{C}\right]$ uracil uptakeas described in Materials and Methods. Results are the average of four measures (two measures in two independent experiments).

\section{Figure 2: Intracellular trafficking of AtUPS1 in yeast.}

A: fur4 $\triangle$ strains transformed with pGAL-UPS1 (triangle) or pGAL-FUR4 (circle) were grown with raffinose as a carbon source. Galactose was then added to induce expression of AtUPS1 and FUR4. The kinetics of plasma membrane delivery of AtUPS1 and Fur4p was determined by quantification of $\left[{ }^{14} \mathrm{C}\right]$ uracil uptake every 30 minutes after galactose induction. Results at each time point are the average of two independent measurements.

B: fur4 $\triangle$ strains transformed with pGAL-UPS1 (triangle) or pGAL-FUR4 (circle) were grown in galactose containing medium. Protein synthesis was inhibited by addition of cycloheximide $(\mathrm{CHX})(100 \mu \mathrm{g} / \mathrm{ml})$. Uracil uptake activity was measured at various time points (two measurements) after $\mathrm{CHX}$ addition. Results are shown as the percentage 
587 Figure 3: Expression of C-terminally GFP tagged AtUPS1 promotes ER 588 proliferation in yeast

589 A: fur4A cells transformed with pGAL, pGAL-UPS1-HA, pGAL-UPS1-GFP and $590 \quad p C Y C 1-U P S 1-G F P$ were tested for growth on plates containing or not 5-fluorouracil $591 \quad(0,75 \mu \mathrm{M})$.

592 B and C: fur4A cells transformed with pCYC-UPS1-GFP, pGAL-UPS1-GFP or pGAL593 Fur4-GFP were grown to mid exponential phase either in glucose, or in raffinose

594 containing medium in the case of strains bearing a plasmid with a GAL promoter. 595 Galactose was then added to induce AtUPS1-GFP or Fur4-GFP expression. At the 596 indicated times, after galactose addition or after growth in glucose containing media 597 (in the case of pCYC-UPS1-GFP), cells were observed by fluorescence microscopy 598 (B) and protein extracts were prepared, resolved by SDS PAGE and analysed by 599 Western immunoblotting using an anti-GFP antibody (C)

600 D: fur4 $\triangle$ cells expressing pGAL and pGAL-UPS1-GFP grown overnight in galactose 601 containing media were processed for electron microscopy. White arrows indicate the 602 endoplasmic reticulum and $\mathrm{N}$ the nucleus.

603

604 Figure 4: Intracellular fate of myc tagged version of AtUPS1

605 A: fur4A cells transformed with pGAL, pGAL-UPS1, and pGAL-UPS1 ${ }^{\text {myc }}$ were tested 606 for 5 -fluorouracil $(1 \mu \mathrm{M})$ sensitivity on plates.

607 B: $\left[{ }^{14} \mathrm{C}\right]$ uracil uptake activity of fur4A cells transformed with pGAL-FUR4, pGAL608 UPS1 and pGAL-UPS1 ${ }^{m y c}$ grown to mid exponential phase on galactose containing 609 medium. Results are the average of two independent measures.

610 C: fur4A strains transformed with pGAL-UPS1 (triangle) or pGAL-UPS1 ${ }^{\text {myc }}$ (square)

611 were grown to mid log phase in raffinose containing medium and galactose was then 
612 added to induce expression of the transporter. The kinetics of plasma membrane

613 delivery of AtUPS1 or Fur4p were determined by quantification of $\left[{ }^{14} \mathrm{C}\right]$ uracil uptake

614 at various times after galactose induction.

615 D: fur4 $\triangle$ strains transformed with pGAL-UPS1 (triangle) or pGAL-UPS1 ${ }^{\text {myc }}$ (square)

616 were grown in galactose containing medium. Uracil uptake activity was measured at 617 different time points after inhibition of protein synthesis by addition of $\mathrm{CHX}$

$618(100 \mu \mathrm{g} / \mathrm{ml})$. Results are shown as the percentage of initial activity.

619 E: WT cells transformed with pGAL-UPS1 ${ }^{\text {myc }}$ were grown to mid log phase in 620 raffinose containing medium. Galactose was then added to induce transporter 621 expression. Protein extracts were prepared at indicated times and proteins were 622 resolved by SDS PAGE and analysed by Western immunoblotting using an anti-myc 623 antibody to detect the transporter and an anti-Gas1p as a loading control.

625 Figure 5: Intracellular localization of UPS $1^{\text {myc }}$ after galactose induction.

626 A: fur4A cells transformed with pGAL-UPS1 $1^{m y c}$ were grown overnight in raffinose 627 containing media. Galactose was added during exponential growth phase. Aliquots 628 were withdrawn at various time points, cells were fixed and processed for 629 immunofluorescence. AtUPS $1^{\text {myc }}$ was detected using an anti-myc antibody as 630 described in Materials and Methods. The nuclei were stained using DAPI. 631 Fluorescence was observed under an Olympus microscope.

632 (Note that, as it is often the case for multicopy plasmid and galactose induction, only 633 a subset of the cells within the population was stained with the anti-myc antibody)

634 B: The same preparations as in (A) were also visualized using confocal microscopy. 635 One section is presented for each time point. 
637 Figure 6: Mutations in RSP5 improve functional expression in yeast of tagged 638 and untagged UPS1

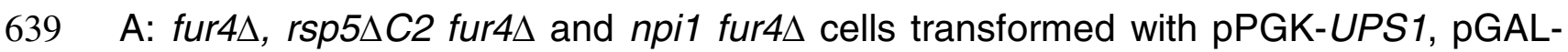
640 UPS1, pGAL-UPS1-GFP or pGAL-UPS1 ${ }^{\text {myc }}$ were grown in glucose containing 641 medium and spotted either on glucose (pPGK-UPS1) or on galactose containing 642 plates (pGAL plasmids) with or without5-fluorouracil as indicated. Different 5FU 643 concentrations were used in order to differentiate optimally the growth of each of the 644 tested strains with regard to the various plasmids: concentration was lower in the 645 case of galactose-induced cells expressing untagged and myc-tagged AtUPS1 that 646 display greater uracil uptake activities than cells expressing GFP-tagged AtUPS1, or 647 untagged AtUPS1 under the control of the PGK promoter. In all cases, growth was 648 tested using a whole range of $5 F U$ concentrations, and a selection of representative 649 plates is shown.

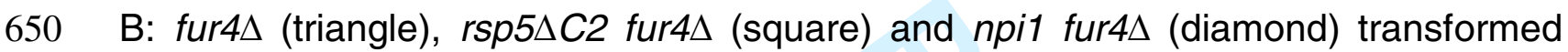
651 with pGAL-UPS1-GFP or pGAL-UPS1 ${ }^{m y c}$ were grown to mid log phase in raffinose 652 containing media and the expression of the various transporters was induced by 653 addition of galactose. Plasma membrane delivery of the transporters was assessed 654 by measuring uracil uptake activity at different time points after galactose addition.

655 C: Induction of AtUPS $1^{\text {myc }}$ was monitored in WT and npi1 cells by Western blot 656 analysis of aliquots withdrawn at several time points after addition of galactose. 657 Proteins were resolved by SDS polyacrylamide gel electrophoresis and analyzed by 658 immunoblotting with anti-myc antibodies for quantification of transporter expression 659 and anti-Gas1p as loading control.

660 
661

662

663

664

665

666

667

668

669

670

671

672

673

674

675

676

677

678

679

680

681

682

683

684

\section{Acknowledgements}

We thank H. Riezman for the kind gift of antibodies, Antonia Kropfinger for editorial assistance, and the members of RHT's laboratory for helpful discussions and comments on the manuscript and. We are grateful to Sophie Le Panse for electron microscopy. We also thank Jennifer Molinari, Youri Lokossa, Ludovic Warroux and Yvonne Sauermann for technical help. This work was supported by a grant to M.D. from the Deutsche Forschungsgemeinschaft (DE 817/1-1). The work done in RHT's lab was supported by the Centre National de la Recherche Scientifique, the Universities Paris 6 and Paris 7, by a grant from the Association pour la Recherche contre le Cancer (ARC) (grant no. 3298), and by an European Union program (EFFEXPORT, contract QLRT-2001-00533). Marine Froissard, Naïma BelgarehTouzé and Jennifer Molinari received fellowships from this program. We are especially indebted to Bruno Andre, coordinator of the EFFEXPORT program, for his constant support.

\section{References}

(1) Schwacke, R.; Schneider, A.; van der Graaff, E.; Fischer, K.; Catoni. E; M., D.; Frommer, W.; Flugge, U.; Kunze, R. Plant Physiol. 2003, 131, 16-26.

(2) Andre, B. Yeast 1995, 11, 1575-611.

(3) Nelissen, B.; De Wachter, R.; Goffeau, A. FEMS Microbiol Rev 1997, 21, 113-34.

(4) Van Belle, D.; Andre, B. Curr Opin Cell Biol 2001, 13, 389-98.

(5) Winzeler, E. A.; Shoemaker, D. D.; Astromoff, A.; Liang, H.; Anderson, K.; Andre, B.; Bangham, R.; Benito, R.;et al. Science 1999, 285, 901-6. 
685 (6) Frommer, W. B.; Ninnemann, O. Annu. Rev. Plant Physiol. Plant Mol. Biol. 1995, 46, 686 419-444.

687 (7) Barbier-Brygoo, H.; Gaymard, F.; Rolland, N.; Joyard, J. Trends Plant Sci 2001, 6, 688 577-85.

689 (8) Dreyer, I.; Horeau, C.; Lemaillet, G.; Zimmermann, S.; Bush, D. R.; Rodriguez690 Navarro, A.; Schachtman, D. P.; Spalding, E. P.; Sentenac, H.; Gaber, R. F. J. Exp. 691 Bot. 1999, 50, 1073-1087.

692 (9) Bush, D. R. Curr Opin Plant Biol 1999, 2, 187-91.

693 (10) Palmgren, M. G.; Christensen, G. J Biol Chem 1994, 269, 3027-33.

694 (11) de Kerchove d'Exaerde, A.; Supply, P.; Dufour, J. P.; Bogaerts, P.; Thines, D.; 695 Goffeau, A.; Boutry, M. J Biol Chem 1995, 270, 23828-37.

696 (12) Niebauer, R. T.; Wedekind, A.; Robinson, A. S. Protein Expr Purif 2004, 37, 134-43.

697 (13) Makuc, J.; Cappellaro, C.; Boles, E. FEMS Yeast Res 2004, 4, 795-801.

698 (14) Wieczorke, R.; Dlugai, S.; Krampe, S.; Boles, E. Cell Physiol Biochem 2003, 13, 12369934.

700 (15) Desimone, M.; Catoni, E.; Ludewig, U.; Hilpert, M.; Schneider, A.; Kunze, R.; 701 Tegeder, M.; Frommer, W. B.; Schumacher, K. Plant Cell 2002, 14, 847-56.

702

(16) Schmidt, A.; Su, Y. H.; Kunze, R.; Warner, S.; Hewitt, M.; Slocum, R. D.; Ludewig, 703 U.; Frommer, W. B.; Desimone, M. J Biol Chem 2004, 279, 44817-24.

704

(17) Haguenauer-Tsapis, R.; André, B. In Control of transmembrane transport; Boles E 705 and Krämer, R., Ed.; Springer Verlag, 2004.

706

(18) Garnier, C.; Blondel, M. O.; Haguenauer-Tsapis, R. Mol Microbiol 1996, 21, 1061-73.

707

(19) Galan, J. M.; Moreau, V.; Andre, B.; Volland, C.; Haguenauer-Tsapis, R. J Biol Chem 708 $1996,271,10946-52$.

709

(20) Rotin, D.; Staub, O.; Haguenauer-Tsapis, R. J Membr Biol 2000, 176, 1-17. 
710 (21) Blondel, M. O.; Morvan, J.; Dupre, S.; Urban-Grimal, D.; Haguenauer-Tsapis, R.; 711 Volland, C. Mol Biol Cell 2004, 15, 883-95.

712

(22) Pizzirusso, M.; Chang, A. Mol Biol Cell 2004, 15, 2401-9.

713

(23) Hoppe, T.; Matuschewski, K.; Rape, M.; Schlenker, S.; Ulrich, H. D.; Jentsch, S. Cell 714 $2000,102,577-86$.

715

716

717

718

719

720

721

722

723

724

725

726

727

728

729

730

731

732

733

734

(24) Rodriguez, M. S.; Gwizdek, C.; Haguenauer-Tsapis, R.; Dargemont, C. Traffic 2003, $4,566-75$.

(25) Hein, C.; Springael, J. Y.; Volland, C.; Haguenauer-Tsapis, R.; Andre, B. Mol Microbiol 1995, 18, 77-87.

(26) Wang, G.; McCaffery, J. M.; Wendland, B.; Dupre, S.; Haguenauer-Tsapis, R.; Huibregtse, J. M. Mol Cell Biol 2001, 21, 3564-75.

(27) Moreau, V.; Galan, J. M.; Devilliers, G.; Haguenauer-Tsapis, R.; Winsor, B. Mol Biol Cell 1997, 8, 1361-75.

(28) Volland, C.; Urban-Grimal, D.; Geraud, G.; Haguenauer-Tsapis, R. J Biol Chem 1994, $269,9833-41$.

(29) Dupre, S.; Haguenauer-Tsapis, R. Mol Cell Biol 2001, 21, 4482-94.

(30) Marchal, C.; Dupre, S.; Urban-Grimal, D. J Cell Sci 2002, 115, 217-26.

(31) Bugnicourt, A.; Froissard, M.; Sereti, K.; Ulrich, H. D.; Haguenauer-Tsapis, R.; Galan, J. M. Mol Biol Cell 2004, 15, 4203-14.

(32) Dupre, S.; Urban-Grimal, D.; Haguenauer-Tsapis, R. Biochim Biophys Acta 2004, $1695,89-111$.

(33) Bachmair, A.; Novatchkova, M.; Potuschak, T.; Eisenhaber, F. Trends Plant Sci 2001, 6, 463-70.

(34) Morvan, J.; Froissard, M.; Haguenauer-Tsapis, R.; Urban-Grimal, D. Traffic 2004, 5, 383-92. 
735 (35) Springael, J. Y.; De Craene, J. O.; Andre, B. Biochem Biophys Res Commun 1999, 257, 561-6.

737

(36) Dunn, R.; Hicke, L. J Biol Chem 2001, 276, 25974-81.

738

(37) Katzmann, D. J.; Sarkar, S.; Chu, T.; Audhya, A.; Emr, S. D. Mol Biol Cell 2004, 15, 739 468-80.

740

(38) Dunn, R.; Klos, D. A.; Adler, A. S.; Hicke, L. J Cell Biol 2004, 165, 135-44.

741

(39) Haynes, C. M.; Caldwell, S.; Cooper, A. A. J Cell Biol 2002, 158, 91-101.

742

743 (40) Soetens, O.; De Craene, J. O.; Andre, B. J Biol Chem 2001, 276, 43949-57.

744 (41) Gietz, D;; St Jean, A.; Woods, R. A.; Schiestl, R. H. Nucleic Acids Res 1992, 20, 745 1425.

746

(42) Wach, A. Yeast 1996, 12, 259-65.

747

(43) Maniatis, T.; Fritsch, E. F.; Sambrook, J. Molecular Cloning: A Laboratory Manual; Cold Spring Harbor Laboratory: Cold Spring Harbor, N.Y., 1982.

749 (44) Cullin, C.; Minvielle-Sebastia, L. Yeast 1994, 10, 105-12.

750 (45) Niedenthal, R. K.; Riles, L.; Johnston, M.; Hegemann, J. H. Yeast 1996, 12, 773-86.

751 (46) Mumberg, D.; Muller, R.; Funk, M. Gene 1995, 156, 119-22.

752 (47) Rentsch, D.; Laloi, M.; Rouhara, I.; Schmelzer, E.; Delrot, S.; Frommer, W. B. FEBS 753 Lett 1995, 370, 264-8.

754 (48) Belgareh-Touze, N.; Avaro, S.; Rouille, Y.; Hoflack, B.; Haguenauer-Tsapis, R. Mol 755 Biol Cell 2002, 13, 1694-708.

756

757 
A
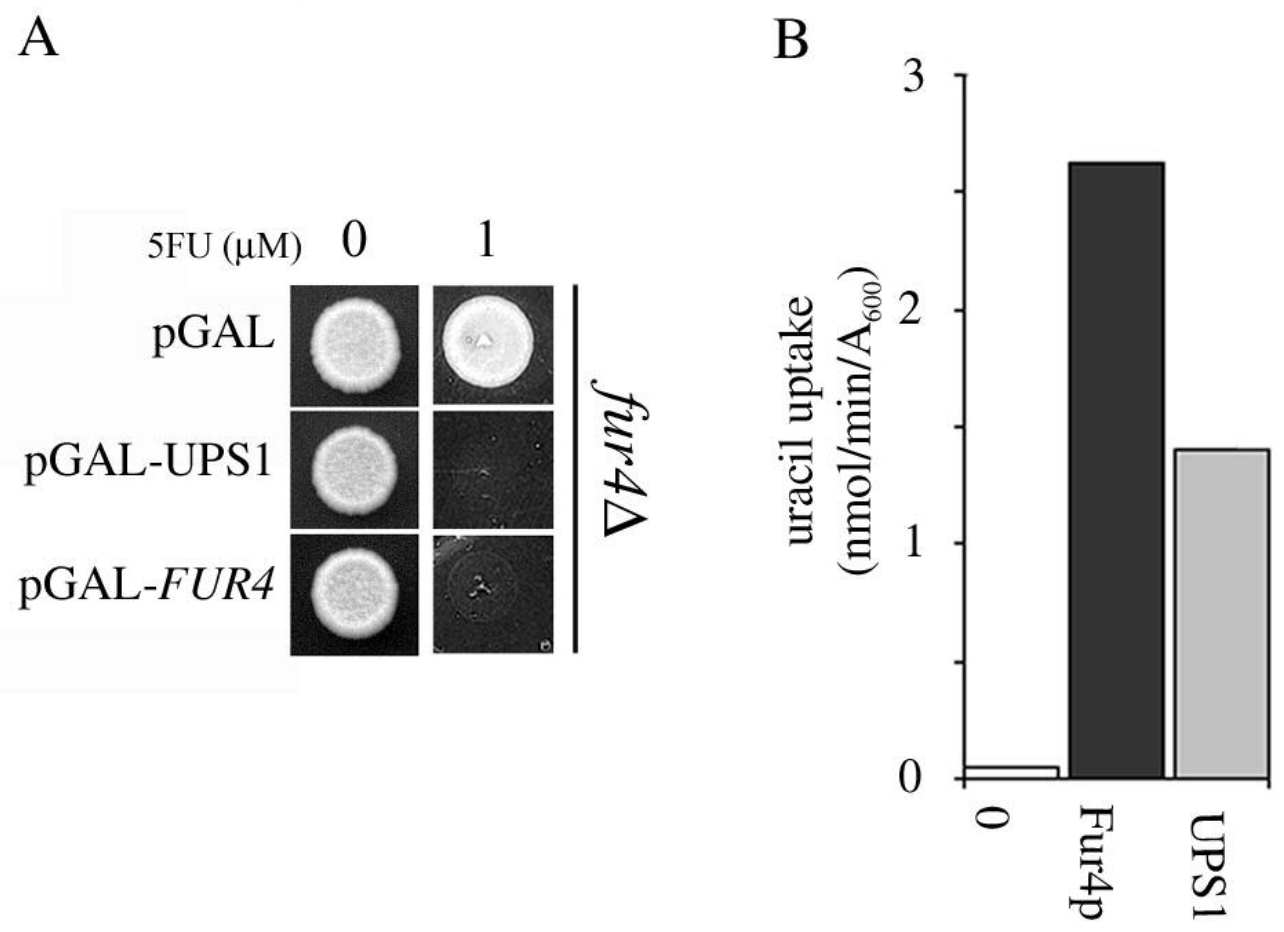
A

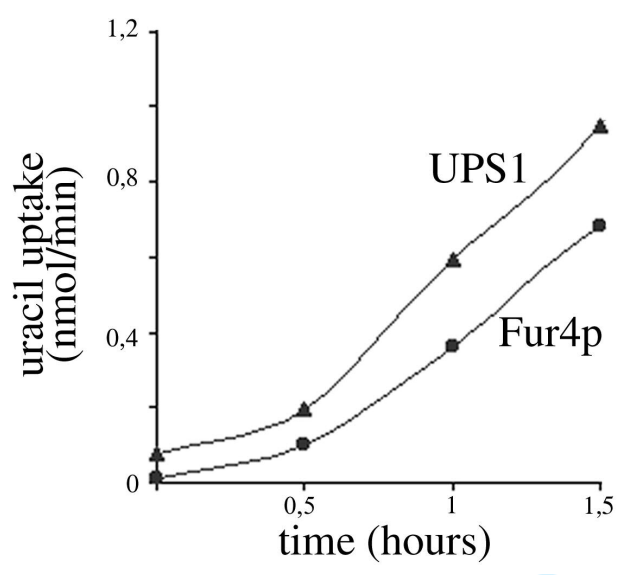

B

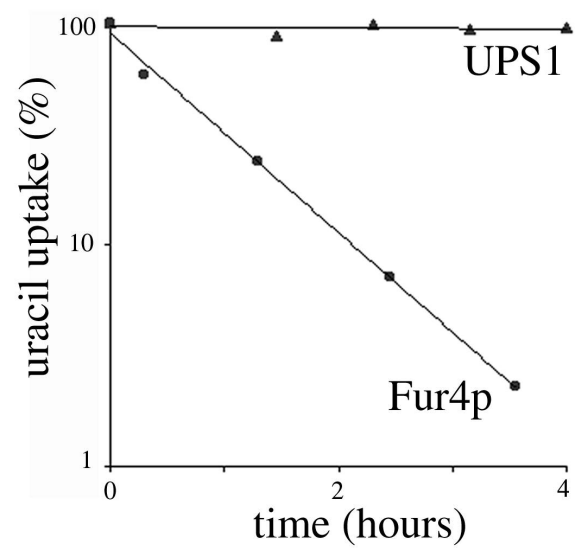




\section{Page 35 of 38}

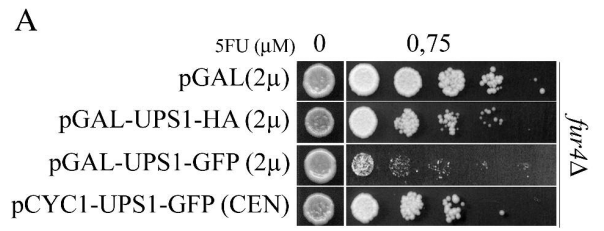

B

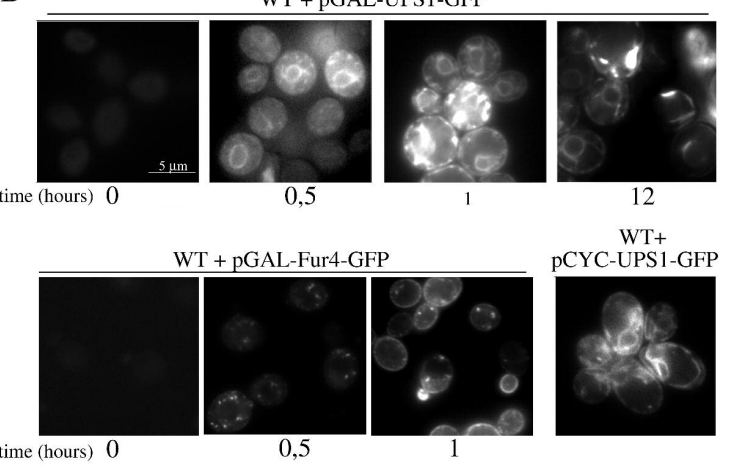

C

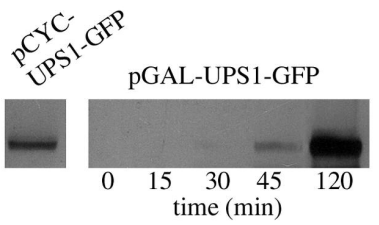

D
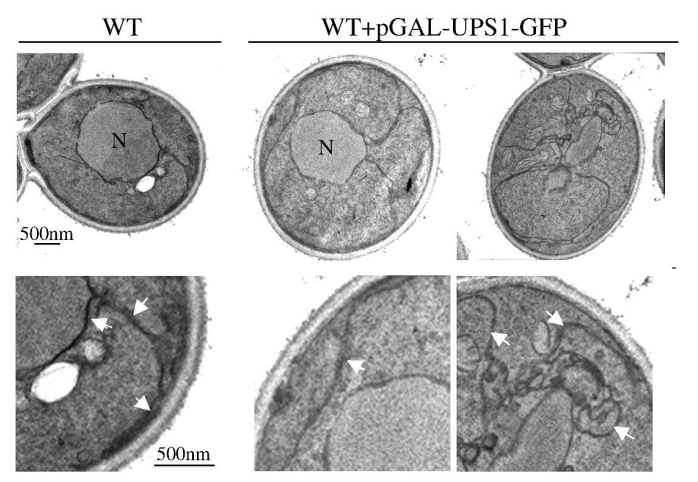
A

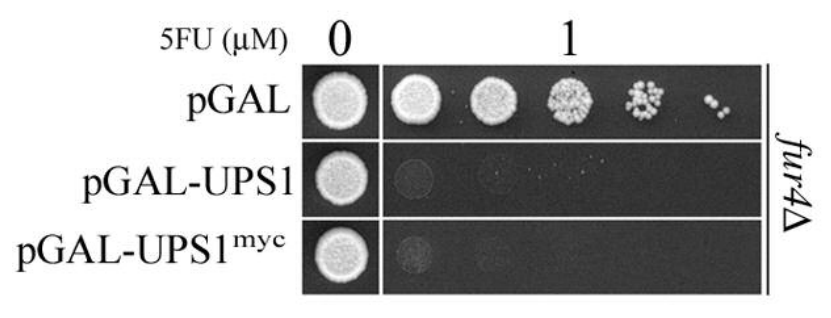

B

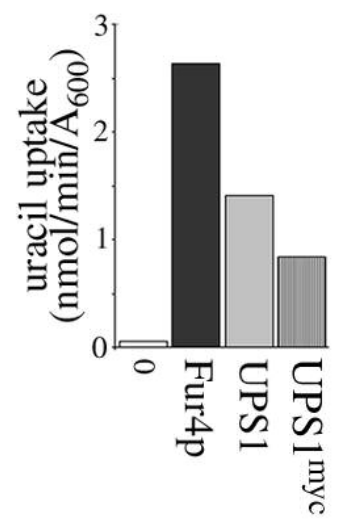

C

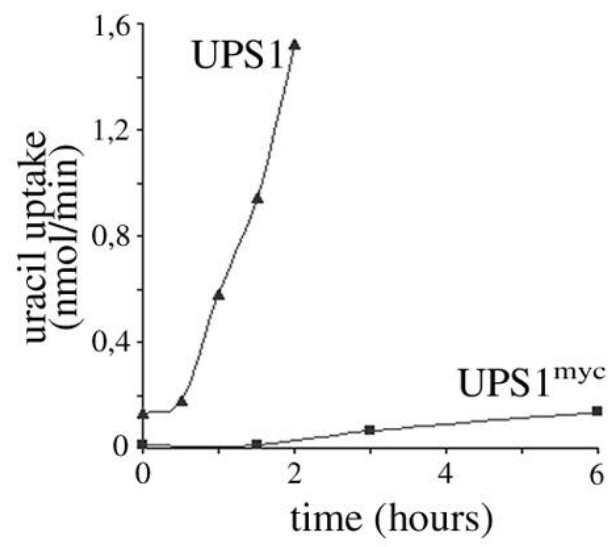

D

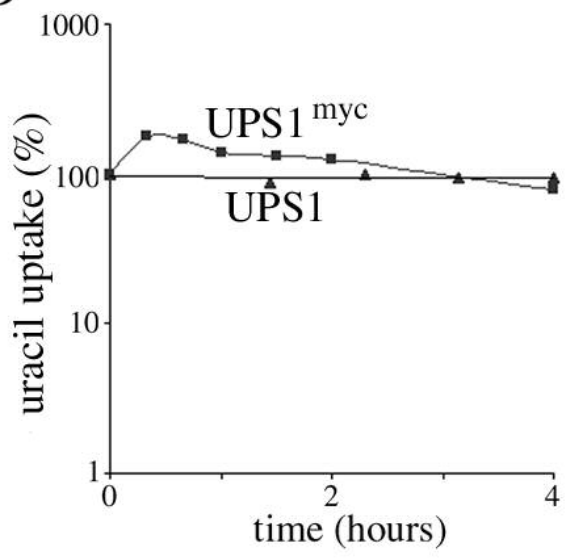

E

time (hours)

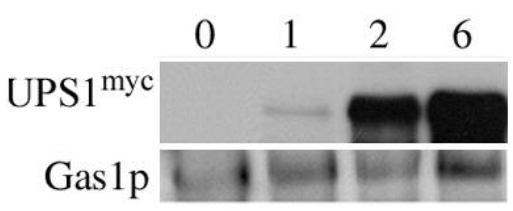


A

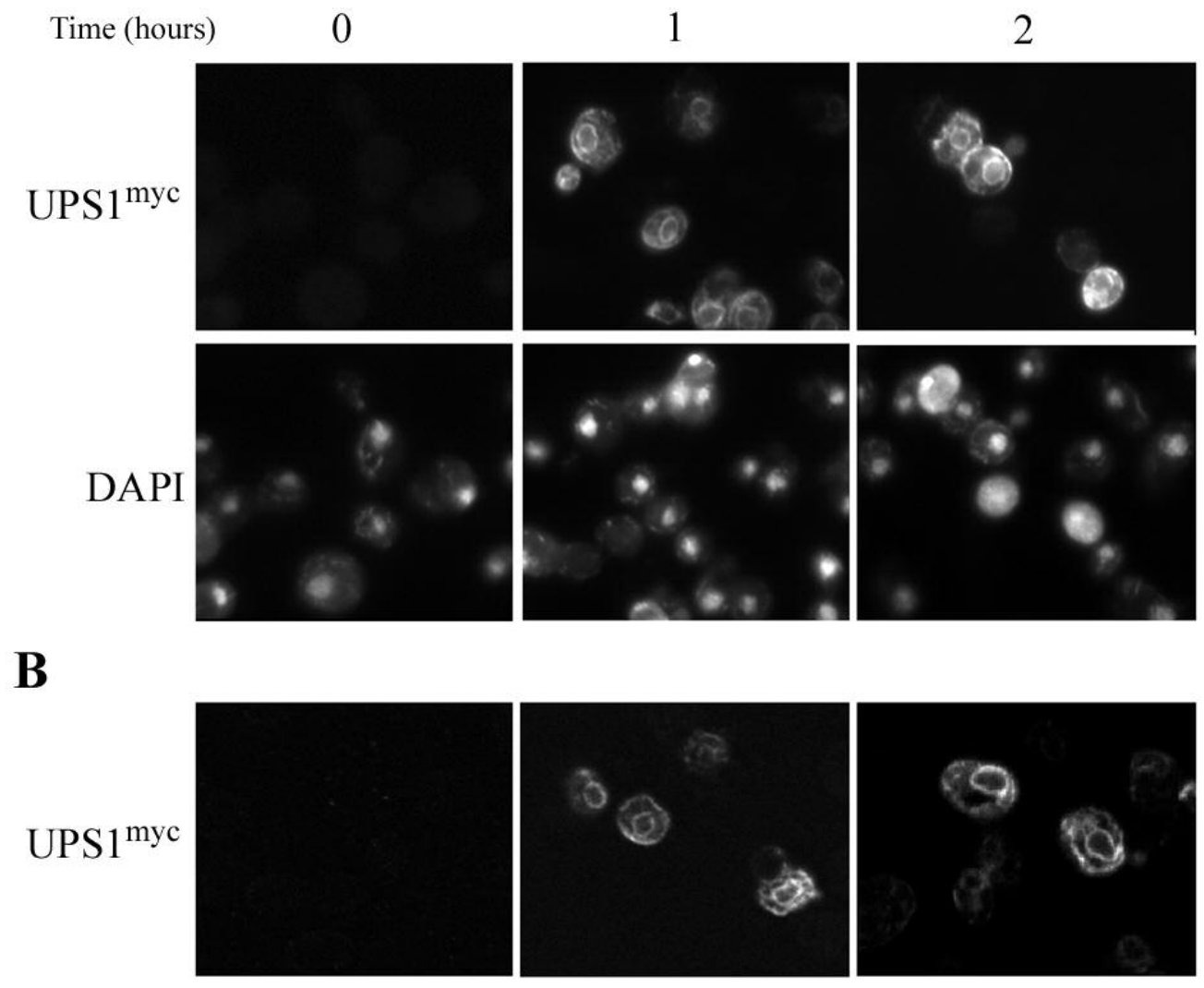


A

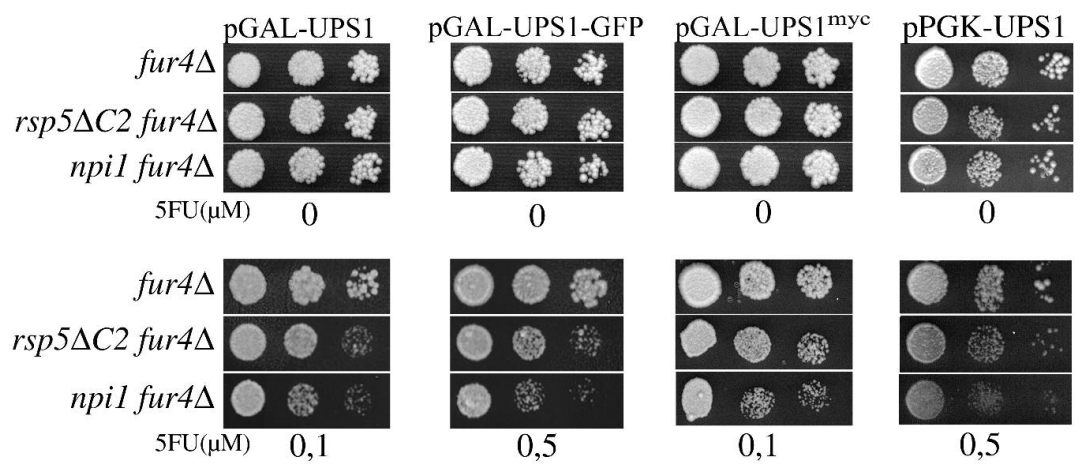

B
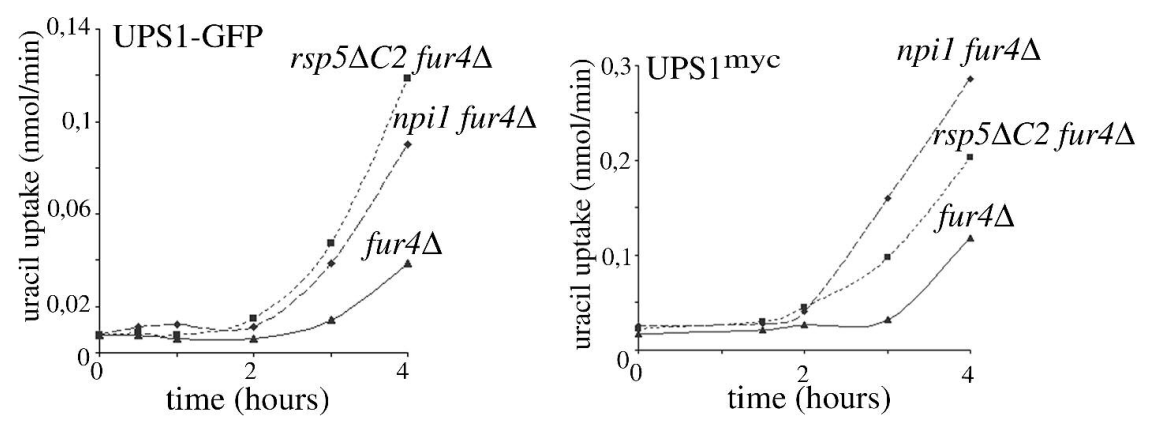

C

$$
\begin{aligned}
& \text { time (hours) }
\end{aligned}
$$

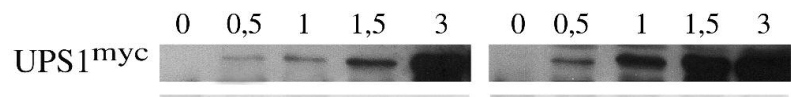

$$
\begin{aligned}
& \text { Gas1 }--\frac{-1}{\text { WT }}-\frac{-1}{n p i l}-
\end{aligned}
$$

